\title{
A System for Recognizing Numeric Strings from Topographical Maps
}

\author{
Masanori ANEGAWA ${ }^{\dagger}$ Osamu SHIKU ${ }^{+\dagger}$ Akira NAKAMURA ${ }^{\dagger}$ \\ Terumitsu OHYAMA $^{+\dagger \dagger} \quad$ Hideo KURODA $^{\dagger}$
}

\author{
+ Dept. of Electrical Engineering and Computer Science, Nagasaki University, Nagasaki 852,JAPAN \\ $+\uparrow$ Sasebo National College of Technology, Nagasaki 857, JAPAN \\ $\dagger_{+}+$Wakayama Shin-Ai Women's Junior College, Wakayama 640, JAPAN
}

\begin{abstract}
This paper proposes a system for recognizing numeric strings from topographical maps, which is composed of the automatic recognition stage and the interactive recognition stage.

In this method, uncertain numeric strings extracted through the automatic recognition stage based on topographical map feature only, are confirmed and corrected by the interactive recognition stage. Therefore we can obtain high precise recognition results.

The method was applied to numeric string recognition from map image which include 102 strings made up of 249 numerals. As a result, 95.1\% of 102 numeric strings were correctly recognized.
\end{abstract}

\section{Introduction}

This paper describes a system for recognizing numeric strings from topographical maps. It is desirable that all the numeric strings are correctly recognized automatically. But there are many problems to make automatic numeric string recognition difficult in topographical maps ${ }^{[1,2]}$.Those problems are :

1) Numerals frequently touch with background figures.

2) There are many numeral-like figures in maps such as a part of roads, symbols or buildings and so on.

This paper describes a system for recognizing numeric strings through the automatic recognition stage and the interactive recognition stage, and its preliminary experiment.

In this system, uncertain numeric strings which are recognized through the automatic recognition stage using topographical map feature only, are confirmed and corrected through the interactive recognition stage. The latter stage checking up on results of the automatic recognition stage, mis-recognized numerals can be corrected. However, the unfound numerals ( which are unfound through the automatic recognition stage) can not be corrected through the interactive recognition stage, because we must search unfound numerals from a vast image.

In this system, in order to reduce the unfound numerals, the pre-reported method ${ }^{[3]}$ for extracting touched characters is adopted, and unfound numerals are supplemented through the re-extraction/recognition process. In addition, in order to detect the unfound numerals easily, the interac- tive recognition stage uses computer display for communication with an operator.

\section{Original Map Image}

A $1 / 50,000$ scale Japanese topographical map print is scanned at a resolution of 320 dots per inch. In this map, numeric strings represent elevation values, and size of a numeral ( $L x \times L y$ pixels) and interval length of both numeral centers ( $L$ pixels) are pre-fixed as shown in Fig. 1.

\section{Numeric String Recognition Method}

Fig. 2 shows the block diagram of the system implementing the method. Fig. 3 demonstrates how to extract and recognize numeric strings.

\subsection{Automatic Recognition Stage}

The automatic recognition stage is composed of four processes; numeral extraction, numeral recognition, grouping, and re-extraction/recognition. The detail is as follows.

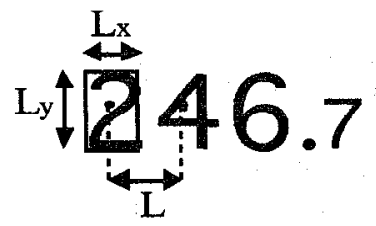

Figure 1 : An example of numeric strings.

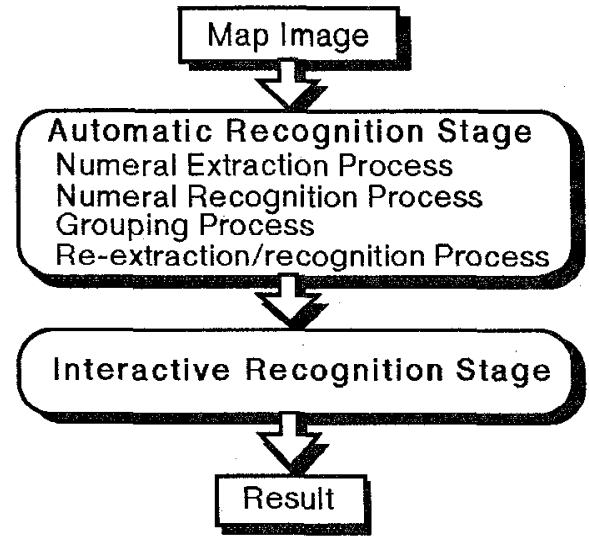

Figure 2 : The system block diagram. 

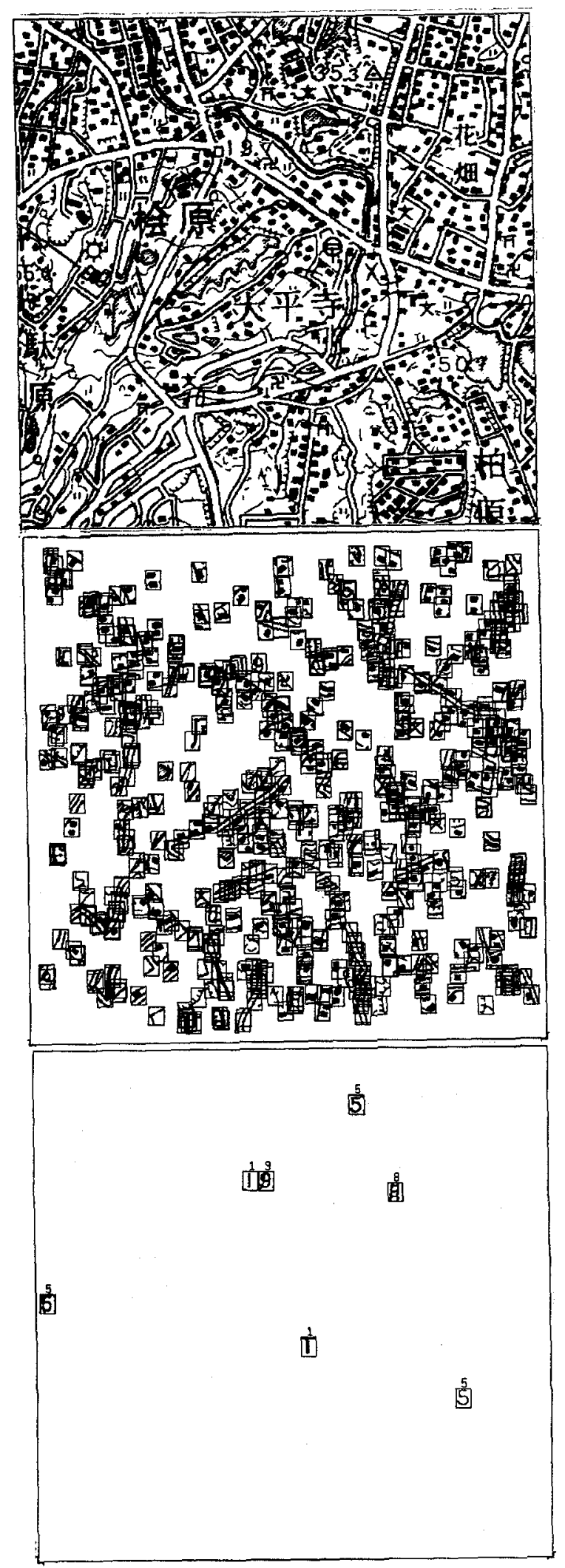

\begin{tabular}{l|l} 
(a) original image $(512 \times 512)$ & (d) results of grouping process
\end{tabular}

(b) resul ts of extraction process (e) results of re-extraction/recognition process (c) results of recognition process (f) displayed recogoition results (g) results of interactive recognition process

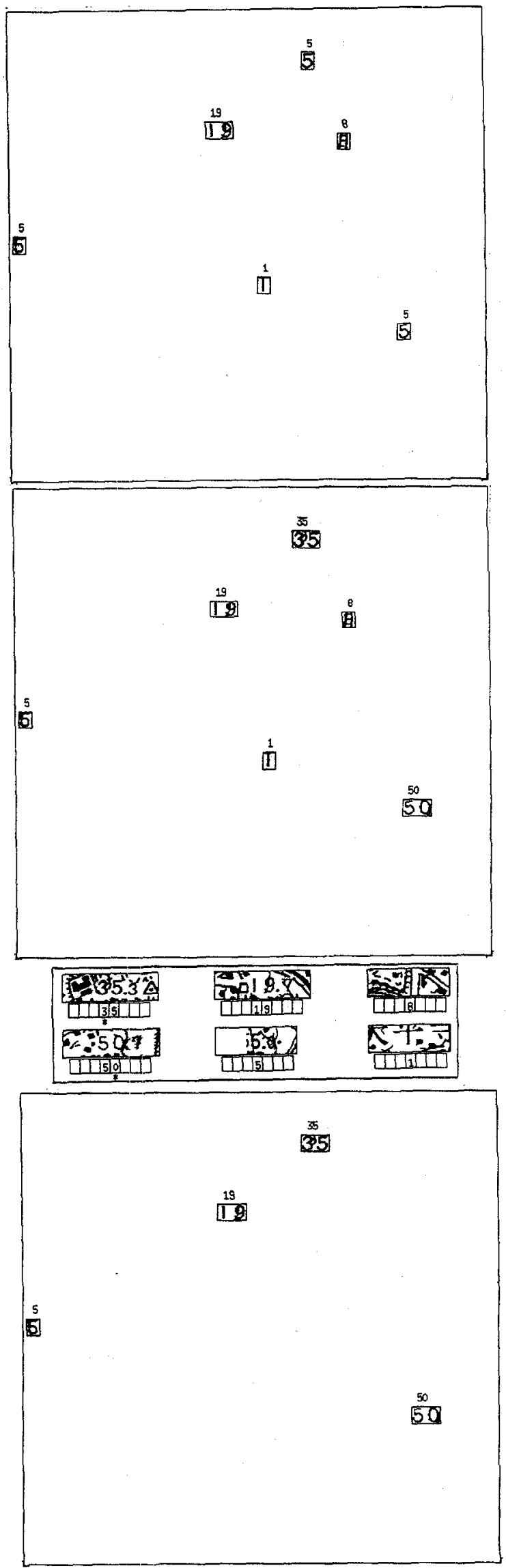

Figure 3 : Dernonstration of numeric string recognition. 


\subsubsection{Numeral Extraction}

Numerals are extracted from a map by the pre-reported character extraction method ${ }^{[3]}$. The extraction algorithm is as follows.

(1) As shown in Fig.4, a prefixed rectangular template ( $L x$ $x L y$ pixels) which is the same size as a numeral, is prepared.

(2) By matching each point on an original binary image with the rectangular template, a black pixel density image is generated (shown in Fig.4). The black pixel density image $g=(g(x, y))$ is given by

$$
\begin{aligned}
& g(x, y)=\frac{\sum_{p \in N} t(p, q) f(x-p, y-q)}{L_{\mathrm{x}} L_{\mathrm{y}}} \\
& \left.N=\left\{\frac{\left[L_{\mathrm{x}}-1\right.}{2}\right] \leq p \leq\left[\frac{L_{\mathrm{x}}}{2}\right],\left[\frac{L_{\mathrm{y}}-1}{2}\right] \leq q \leq\left\lfloor\frac{L_{\mathrm{y}}}{2}\right]\right\} \\
& {[\mathrm{]}: \text { Gaussian symbol, }}
\end{aligned}
$$

where $t=(t(x, y))$ is a rectangular template and $f=(f(x, y))$ is an original binary image.

(3) In order to extract the center point of numerals, the following three conditions for the black pixel density $g(x, y)$ are introduced.

Condition 1 : Density $g(x, y)$ is the greatest in $3 \times 3$ or $5 \times 5$ neighborhoods of the target center point.

Condition 2: $\alpha_{\min }<g(x, y)<\alpha_{\max }$

Condition $3: g^{\prime}(x, y) / g(x, y)<\beta$

where $\alpha_{\min }$ and $\alpha_{\max }$ are the lower and upper bounds for $g(x, y)$ that affect the allowance of pseudo numerals, and $g^{\prime}(x, y)$ is the density for the enlarged template $t^{\prime}$ of $L x^{\prime} \times$ $L y^{\prime}$ pixels shown in Fig.5. Condition 3 decides allowance for noise around the target numerals.

The points which satisfy all the above three conditions, are regarded as the numeral center positions.

(4) Rectangular domains ( $L x \times L y$ pixels) are forcibly extracted as numeral candidate domains from the original image, regarding the above numeral center positions as rectangular domain center positions.

By this method, we can extract correctly even such numerals as touching with background figures. But, at the same time, many pseudo numerals are also extracted (shown in Fig.3(b)).

\subsubsection{Numeral Recognition}

Through the numeral recognition process, true numerals are taken out from the extracted numeral candidates.

In this process, to reduce matching cost, the numeral candidates are limited with pre-classification using rough numeral features ( for example, line width, black pixels distribution and so on ).

Next, the limited candidates are compared with all the standard numerals registered in a numeral dictionary. Comparison algorithm is based on the similarity of $5 \times 5$ mesh features ${ }^{[4]}$ as shown in Eq.(3).

$$
\rho_{c}=\left(\boldsymbol{X}, \boldsymbol{Y}_{c}\right) /\|\boldsymbol{X}\|\left\|\boldsymbol{Y}_{c}\right\|
$$

$X$ : mesh feature for unknown rectangular figure.

$Y_{c}:$ mesh feature for standard numeral pattern.

$$
\text { ( } c \text { :category) }
$$

If the similarity $\rho_{c}$ between an unknown figure and one of the registered standard numerals is greater than the prefixed threshold $\rho_{\mathrm{T} 1}$, the compared unknown figure is regarded as a true numeral.

It is difficult to recognizing "1" using above method, because numeral " 1 " is a very simple structure. Then, the figure of which vertical length is $(L y \pm 1)$ pixels and width is under 3 pixels, is regarded as " 1 ".

If the recognized numerals are overlapped, a numeral of the local maximum similarity is reserved and other numerals are deleted.

Fig.3(c) shows the recognized results corresponding with Fig.3 (b). Almost numeral-like figures were deleted, but a few true numerals were unfound and some numerallike figures were over-recognized.

\subsubsection{Grouping}

The recognized numerals which are placed in horizontal at intervals of $L$ pixels, are grouped into numeric strings ( for example, "19" in Fig.3(d) ).

\subsubsection{Re-extraction/recognition}

A few numerals that touch with heavy background noise are sometimes remained unfound. For these unfound numerals, the re-extraction/recognition process is carried out.

As shown in Fig.6, rectangular domains $(L x \times L y)$ are forcibly extracted from the original image, regarding all the points in the regions ( $3 \times 7$ pixel size dot area in Fig.6) adjacent to the previous recognized numeric string by distance $L$ pixels as the center points of domains. And each rectangular domain is compared with the standard numerals. If the similarity is greater than the pre-fixed threshold $\rho_{\mathrm{T} 2}$, the rectangular domain is regarded as a true numeral ( for example, "35", "50" in Fig.3(e) ).
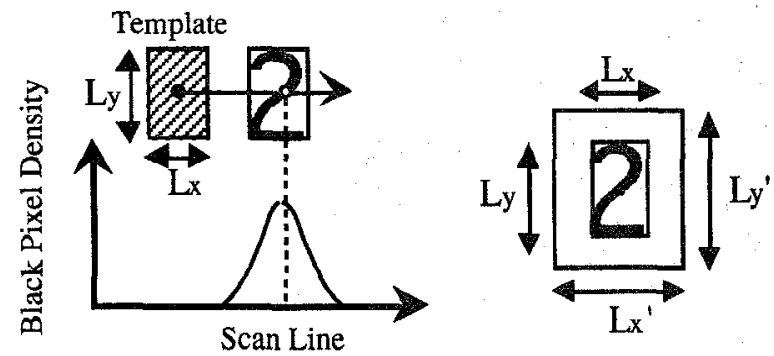

Figure 4:Template and black Figure 5:Enlarged template. pixel density.

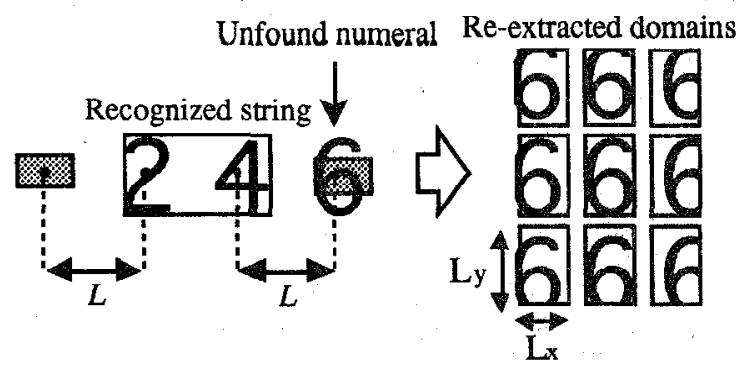

Figure $6:$ Re-extraction / recognition. 


\subsection{Interactive Recognition Stage}

In the interactive recognition stage, the results of the automatic recognition stage are confirmed, then mis-recognized numerals are corrected and unfound numerals are inputted by an operator.

The recognized strings and the string areas on the original image are displayed simultaneously as shown in Fig.3(f). Because the displayed area is wider than the recognized string area, even if only one numeral in a string is found correctly through the automatic recognition stage, the whole string is displayed. Thus, the operator can find the unfound numerals in the numeric string (for example, "1" or "8" adjacent to "9" in Fig.7), and judge easily whether the displayed results are true or false (for example, "1" in Fig.7).

The operator takes out true numeric strings from the displayed results of automatic recognition stage. And recognition errors are corrected. Because the operator pays attention to the displayed results only, the input labor is saved. Fig.3(g) shows the final results corresponding with Fig.3(a).

\section{Preliminary Experiment Result and Discussion}

In order to estimate performance of the proposed system, a preliminary experiment was carried out for original map image (a 50,000:1 topographical map of Sasebo area ; $5120 \times 3740$ pixels) which involved 102 numeric strings (249 numerals).

Table 1 shows the experimental parameters. $\rho_{\mathrm{T} 1}$ and $\rho_{\mathrm{T} 2}$ are experientially settled, and other parameters are settled by map drawing rule and input resolution.

The results of the experiment are as follows. About 590 rectangular domains per true numeral were extracted as numeral candidates from the original image through the numeral extraction process. Those rectangular domains were reduced to about 1.7 candidates per correct recognized numeral through the numeral recognition process. The fact shows that several non-numeral figures are picked up as numerals in the numeral recognition process, in spite of rather severe threshold value of $\rho_{\mathrm{T} 1}$. And recognition rate remains low level of $80.7 \%$. From these results of numeral recognition process, the $64.7 \%$ of 102 true strings were correctly grouped in the grouping process. In addition, through the re-extraction/recognition process, the numeral recognition rate increases from $80.7 \%$ to $90.8 \%$, and the numeric string recognition rate increases from $64.7 \%$ to $80.4 \%$.

The probability of finding at least one numeral in a string through the automatic recognition stage, that is, the probability that a string is displayed correctly in the interactive recognition, was $95.1 \%$. Therefore, $95.1 \%$ of 102 strings were correctly recognized in the proposed system, which consists of the automatic recognition stage and the interactive recognition stage.

At the same time, about 0.7 non-numeric strings per correct displayed string were over-recognized. Especially, it is remarkable that simple shape numeral ("1") was often over-recognized.

Fig. 8 shows examples of experimental results. Fig.8(a) shows examples of displayed numeric strings in the interactive recognition stage. Fig.8(b) shows examples of unfound (non-displayed) numeric strings. In Fig.8(a) symbol * shows a supplemented numeral. Unfound numerals are due to touching or scratching or crushing noise.

Table 1 The experimental parameters.

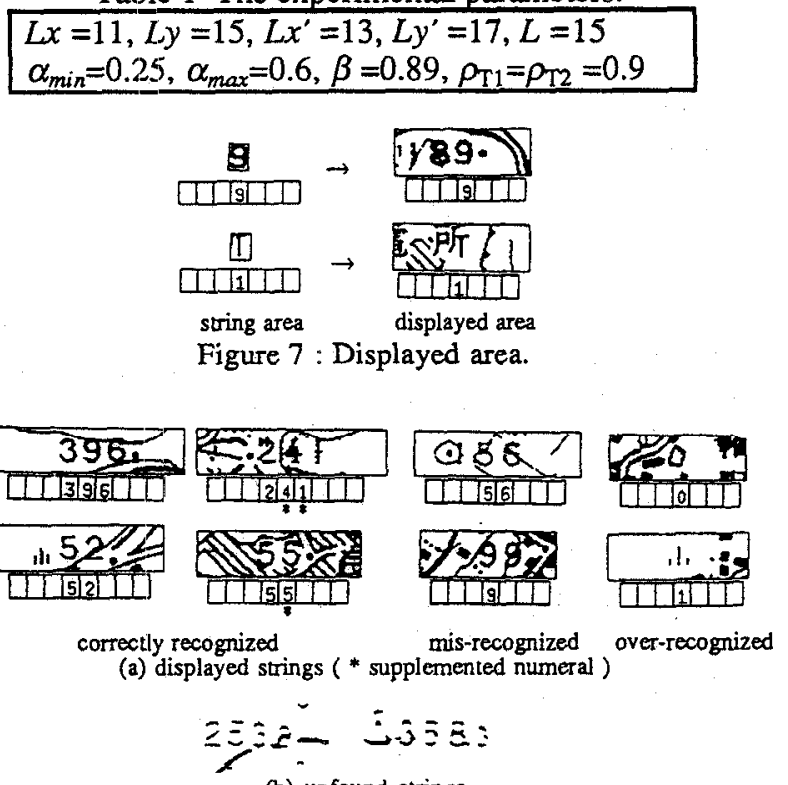

Figure 8 : Examples of experimental results.

\section{Conclusion}

This paper proposed a system for recognizing numeric strings from maps, which was composed of the automatic recognition stage and the interactive recognition stage.

In this system, uncertain numeric strings extracted through the automatic recognition stage based on topographical map feature only, are confirmed and corrected by the interactive recognition stage. Therefore we can obtain high precise recognition results.

The system was applied to string recognition from map image which include 102 strings made up of 249 numerals. As a result, $95.1 \%$ of 102 strings were correctly recognized.

References

[1] Yamamoto,K., Yamada,H. and Murai,S., "Symbol recognition and surface recostruction from topographic map by parallel method", Proc. 2nd ICDAR, pp.914-917, 1993 [2] Nakamura,A., Shiku,O., Anegawa,M., Nakamura,C. and Kuroda,H., "A Method for Recognizing Character Strings from Maps Using Linguistic Knowledge", Proc. 2nd ICDAR, pp561 $-564,1993$.

[3] Shiku, O., Anegawa,M., Nakamura,C. and Nakamura,A., " Character extraction from map image ", Tran, JPS Japan, vol. 34, pp. 273-280, Feb. 1993.

[4] Meguro,S. and Umeda,M. "Recognition of multi-font Chinese characters," Tran, Inst. Elec. Inf. Com. Eng. Japan , J65-D, pp, 1026-1033, Aug. 1982. 